\title{
Disinhibition bursting of dopaminergic neurons
}

\author{
Collin J. Lobb, Todd W. Troyer, Charles J. Wilson and Carlos A. Paladini*
}

Neurosciences Institute, University of Texas at San Antonio, San Antonio, TX, USA

\section{Edited by:}

James M. Tepper, Rutgers, The State

University of New Jersey, USA

Reviewed by:

Mark Bevan, Northwestern University, USA

Mark D. Humphries, University of Sheffield, UK

\section{${ }^{*}$ Correspondence:}

Carlos A. Paladini, Neurosciences Institute, University of Texas at San Antonio, One UTSA Circle,

San Antonio, TX 78249, USA.

e-mail: carlos.paladini@utsa.edu
Substantia nigra pars compacta (SNpc) dopaminergic neurons receive strong tonic inputs from GABAergic neurons in the substantia nigra pars reticulata (SNpr) and globus pallidus (GP), and glutamatergic neurons in the subthalamic nucleus. The presence of these tonic inputs raises the possibility that phasic disinhibition may trigger phasic bursts in dopaminergic neurons. We first applied constant NMDA and GABA ${ }_{A}$ conductances onto a two-compartment single cell model of the dopaminergic neuron (Kuznetsov et al., 2006). The model exhibited disinhibition bursting upon stepwise removal of inhibition. A further bifurcation analysis suggests that disinhibition may be more robust than excitation alone in that for most levels of NMDA conductance, the cell remains capable of bursting even after a complete removal of inhibition, whereas too much excitatory input will drive the cell into depolarization block. To investigate the network dynamics of disinhibition, we used a modified version of an integrate-and-fire based model of the basal ganglia (Humphries et al., 2006). Synaptic activity generated in the network was delivered to the two-compartment single cell dopaminergic neuron. Phasic activation of the D1-expressing medium spiny neurons in the striatum (D1STR) produced disinhibition bursts in dopaminergic neurons through the direct pathway (D1STR to SNpr to SNpc). Anatomical studies have shown that D1STR neurons have collaterals that terminate in GP. Adding these collaterals to the model, we found that striatal activation increased the intra-burst firing frequency of the disinhibition burst as the weight of this connection was increased. Our studies suggest that striatal activation is a robust means by which disinhibition bursts can be generated by SNpc dopaminergic neurons, and that recruitment of the indirect pathway via collaterals may enhance disinhibition bursting.

Keywords: dopamine, GABA, burst, model, network

\section{INTRODUCTION}

In behaving animals, midbrain dopaminergic neurons fire bursts of action potentials in response to salient stimuli (Redgrave et al., 1990; Horvitz et al., 1997; Schultz, 1998), when a greater than expected reward is received (Schultz et al., 1997), or during sequence learning (Jin and Costa, 2010). The mechanisms by which dopaminergic neurons generate bursts are an area of intense study. The absence of burst firing in slices (e.g., Kita et al., 1986; Grace and Onn, 1989) suggests that bursts are generated in response to afferent input. One view of the dopaminergic neuron in vivo is that single-spiking is generated by an in vitro-like calcium - (Kita et al., 1986; Grace and Onn, 1989; Wilson and Callaway, 2000) or sodium-based (Guzman et al., 2009; Khaliq and Bean, 2010) pacemaking mechanism in which spikes can be advanced or delayed due to afferent input. Under such a scheme, bursts are caused by phasic excitatory inputs, e.g., inputs activating AMPA, NMDA, or nicotinic acetylcholine receptors (Grace and Bunney, 1984; Grenhoff et al., 1986; Chergui et al., 1993; Morikawa et al., 2003; Blythe et al., 2007, 2009; Deister et al., 2009; Lobb et al., 2010). However, dopaminergic neurons in vivo are subject to strong tonic synaptic inputs, suggesting that an alternative but complementary mechanism by which dopaminergic neurons may fire bursts is through disinhibition.

Dopaminergic neurons receive strong synaptic activation from spontaneously active inputs in vivo (e.g., Grace and Bunney, 1985; Tepper and Lee, 2007). The effect of these tonic inputs can be seen by subtraction. Local application of NMDA, but not AMPA, receptor antagonists significantly reduce spontaneous burst firing in vivo (Charlety et al., 1991; Overton and Clark, 1992; Chergui et al., 1993; Christoffersen and Meltzer, 1995). Paladini and Tepper (1999), and Brazhnik et al. (2008) found that local application of a variety of GABA antagonists by pressure ejection onto the recorded neuron shifted the firing pattern of the dopaminergic neuron from a single-spiking mode to a bursting one, indicating that tonic $\mathrm{GABA}_{\mathrm{A}}$ receptor activation was strong enough to suppress burst firing. These experiments suggest that dopaminergic neurons are subject to at least two types of tonic inputs in vivo: an NMDA-mediated excitatory input and a $\mathrm{GABA}_{\mathrm{A}}$-mediated inhibitory input. We have recently demonstrated that dopaminergic neurons that receive tonic NMDA and $\mathrm{GABA}_{\mathrm{A}}$ inputs can maintain their spontaneous activity and are capable of generating bursts through disinhibition (Lobb et al., 2010). Using a combination of network and compartmental computational modeling, we explore how disinhibition bursting of dopaminergic neurons might arise from striatal activation.

\section{MATERIALS AND METHODS}

This study investigated conditions under which disinhibition in the basal ganglia network leads to bursting in midbrain dopaminergic neurons. Our model was based on a combination of two previously published models. The first is the current-based, integrate-and-fire type network model of the basal ganglia described in Humphries et al. (2006). This network model was used to generate the synaptic input onto a two-compartment model of a substantia nigra pars compacta (SNpc) dopaminergic neuron (Kuznetsov et al., 2006). 
The network model contained five collections of neurons: D1STR (D1-expressing striatum, $N=64$ neurons, threshold $\theta=30 \mathrm{mV}$, time constant $\tau_{\mathrm{m}}=25 \mathrm{~ms}$ ), globus pallidus (GP; $N=64, \theta=30 \mathrm{mV}$, $\left.\tau_{\mathrm{m}}=14 \mathrm{~ms}\right), \operatorname{STN}\left(N=64, \theta=20 \mathrm{mV}, \tau_{\mathrm{m}}=6 \mathrm{~ms}\right)$, substantia nigra pars reticulata $\left(\mathrm{SNpr} ; N=64, \theta=30 \mathrm{mV}, \tau_{\mathrm{m}}=8 \mathrm{~ms}\right.$ ), and $\mathrm{SNpc}$ $(N=64$, see single cell model below). See Humphries et al. (2006) for the full specification of the model.

The original Humphries et al. (2006) model consists of multiple anatomically based microchannels to allow for a mechanism of action selection. For simplicity, we employed only a single microchannel. Connections were added between nuclei as shown in Figure 1. The D1STR-SNpc connection arises from neurons in striatal patches whereas the D1STR-SNpr connection arises from neurons in the striatal matrix (Gerfen, 1984). It is unclear if both would be activated during a rewarding scenario and thus the D1STR-SNpc connection is disregarded in this study. All connections were given a weight of $-1,0$, or +1 , where a negative value represents an inhibitory connection and a positive value represents an excitatory connection. Connections could be removed or disabled in the network by setting the connection weight to zero. An afferent neuron made a synaptic connection onto each neuron in a target nucleus with a probability of 0.25 . As described in Humphries et al. (2006), inhibitory connections were modeled as exponentially decaying negative currents with a decay time $\tau_{\mathrm{GABA}}=3 \mathrm{~ms}$. Excitatory connections had both AMPA and NMDA components, each modeled as a simple decaying exponential with decay times $\tau_{\mathrm{AMPA}}=2 \mathrm{~ms}$ and $\tau_{\mathrm{NMDA}}=100 \mathrm{~ms}$. The peak amplitude of individual AMPA and $\mathrm{GABA}_{\mathrm{A}}$ postsynaptic potentials were $3 \mathrm{mV}$; individual NMDA potentials peaked at $0.1 \mathrm{mV}$. Dopamine levels were set to the "normal" condition in Humphries et al. $\left(2006 ; \lambda_{\mathrm{D} 1}=\lambda_{\mathrm{D} 2}=0.3\right)$. Background pacemaking activity seen in basal ganglia neurons (STN: $12 \mathrm{~Hz}, \mathrm{GP}: 26 \mathrm{~Hz}$, SNpr: $32 \mathrm{~Hz}$ ) was induced by inject-



FIGURE 1 | Circuit diagram. The six nuclei and their interconnections adapted from the Humphries et al. (2006) network model of the basal ganglia. The SNpc was added. The connections shown in gray are described in the original model while those in blue are added here. The connection from D1STR to the GP was added in accordance with anatomical data of Kawaguchi et al. (1990). ing a suprathreshold fixed current. The level of this current was adjusted to maintain background firing rates for the reduced model described in Figures 3-5 (STN: 66.1 pA, GP: 17 pA, SNpr: 14 pA) and the full model described in Figures 6 and 7 (STN: 100.5 pA, GP: 17 pA, SNpr: 26 pA). As described in Humphries et al. (2006), each neuron model contained a noise term with a mean \pm SD of $0 \pm 0.0008 \mathrm{mV}$.

Disinhibition bursts were initiated by modeling bursts of cortical inputs as step pulses of current delivered to D1STR neurons $(+2 \mathrm{nA}, 150 \mathrm{~ms})$. Simulations were run for $1 \mathrm{~s}$ before these current injections to mitigate any effect of onset transients. Simulations were implemented in MATLAB (Mathworks, Natick, MA, USA) as described in Humphries et al. (2006).

Inputs to the single cell SNpc model arose from the SNpr, GP, and STN. The SNpr and GP inputs made synapses onto both somatic and dendritic compartments of the two-compartment model and activated postsynaptic $\mathrm{GABA}_{\mathrm{A}}$ receptors on dopaminergic neurons (Paladini and Tepper, 1999). STN inputs made synapses onto the burst-generating, dendritic compartment of the two-compartment model and activated postsynaptic NMDA receptors. Postsynaptic activation of AMPA receptors was excluded because local application of an AMPA receptor antagonist in vivo does not change the activity of dopaminergic neurons (Chergui et al., 1994). In contrast, tonic STN input contributes to spontaneous bursting in vivo (Smith and Grace, 1992; Chergui et al., 1994) that is strongly affected by local application of NMDA receptor antagonists (Chergui et al., 1994). $\mathrm{GABA}_{\mathrm{A}}$-mediated currents were added to both somatic and dendritic compartments of the two-compartment dopaminergic neuron model.

The synaptic conductances waveforms were described as a difference of exponentials: $G \operatorname{syn}(t)=\delta *\left[\mathrm{e}^{\left(-t / \tau_{\text {fall }}\right)}-\mathrm{e}^{\left(-t / \tau_{\text {rise }}\right)}\right]$. Spike times were drawn from activation in the network model and each triggered a step increase of size $\delta$. For GABA receptors (input from $\mathrm{GP}$ and $\mathrm{SNr}), \tau_{\text {rise }}=1 \mathrm{~ms}, \tau_{\text {fall }}=6 \mathrm{~ms}$, and $\delta_{\mathrm{GABA}}=0.043 \mathrm{~ms} / \mathrm{cm}^{2}$. For NMDA receptors (input from STN), $\tau_{\text {rise }}=3 \mathrm{~ms}, \tau_{\text {fall }}=40 \mathrm{~ms}$, and $\delta_{\mathrm{NMDA}}=0.030 \mathrm{~ms} / \mathrm{cm}^{2}$.

Single cell simulations were run in XPPAUT (Ermentrout, 2002) using the RUNGE-KUTTA method with $\mathrm{d} t=0.01$ or $0.005 \mathrm{~ms}$. Bifurcation diagrams were made with the XPPAUT implementation of AUTO. Equations were implemented as described in the original model and are not reproduced here. The full set of parameters used here is given in Table 1. Calcium buffering parameters are identical to the original model with the exception of the calcium pump density, which was increased from 2500 to $10,000 \mu \mathrm{m} / \mathrm{s}$ to increase the frequency of dopaminergic neuron bursting produced in the model.

Table 1 | Parameters used in the compartmental model.

\begin{tabular}{llllll}
\hline Parameter & $\begin{array}{l}\text { Value } \\
\left(\mathbf{m s} / \mathbf{c m}^{2}\right)\end{array}$ & Parameter & Value & Parameter & Value \\
\hline$g_{\text {Leak }}$ & 0.095 & $g_{\mathrm{NMDA}}$ & See text & $E_{\text {Leak }}$ & $-50 \mathrm{mV}$ \\
$g_{\mathrm{KCa}}$ & 0.3 & $g_{\mathrm{GABAA}}$ & See text & $r 1$ & $10 \mu \mathrm{m}$ \\
$g_{\mathrm{K} 2}$ & 10 & $E_{\mathrm{Na}}$ & $55 \mathrm{mV}$ & $r^{2}$ & $0.5 \mu \mathrm{m}$ \\
$g_{\mathrm{Na}}$ & 150 & $E_{\mathrm{Ca}}$ & $100 \mathrm{mV}$ & $n_{\mathrm{d}}$ & 10 \\
$g_{\mathrm{K} 1}$ & 0.0 & $E_{\mathrm{K}}$ & $-90 \mathrm{mV}$ & $\mathrm{Mg}$ & $0.5 \mathrm{mM}$ \\
$g_{\mathrm{C}}$ & 0.25 & $E_{\mathrm{NMDA}}$ & $0 \mathrm{mV}$ & $V_{\mathrm{SK}}$ & 7 \\
$g_{\mathrm{Ca}}$ & 0.15 & $E_{\mathrm{GABAA}}$ & $-60 \mathrm{mV}$ & $V_{\mathrm{HK}}$ & $-10 \mathrm{mV}$
\end{tabular}




\section{RESULTS \\ SINGLE CELL}

We first investigated the range of synaptic parameters that can give rise to bursting in our single cell model of the midbrain dopaminergic neuron. When all the synaptic conductances are set to 0 , the dopaminergic neuron shows a very regular pattern of spiking at a frequency of approximately 4-5 Hz (Figure 2A). The model can generate a sustained burst in response to a phasic NMDA conductance (Figure 2B, $g_{\mathrm{NMDA}}=0.28 \mathrm{~ms} / \mathrm{cm}^{2}$ at $1100 \mathrm{~ms}$ ). Bursts could also be generated through disinhibition. Constant NMDA and GABA conductances were applied to the model to maintain balanced spiking (Figure 2C; $g_{\mathrm{GABAA}}=0.14 \mathrm{~ms} / \mathrm{cm}^{2}, g_{\mathrm{NMDA}}=0.28 \mathrm{~ms} / \mathrm{cm}^{2}$ ). At $t=1100 \mathrm{~ms}$ the $\mathrm{GABA}_{\mathrm{A}}$ conductance was set to 0 and the cell fired a sustained burst of action potentials (intra-burst frequency in Figure 2B,C are both $47 \mathrm{~Hz}$ ).

Given that dopamine neuron bursting can result from either an increase in excitation or a removal of inhibition, we determined which combinations of $\mathrm{GABA}_{\mathrm{A}}$ and NMDA conductance values permitted repetitive spiking. In particular, we calculated a twoparameter bifurcation diagram that separated oscillatory (repetitive spiking) behavior from fixed-point (non-spiking) behavior (Figure 2D). The hatched region represents combinations of syn- aptic conductances for which the cell fires trains of action potentials. Parameters below to the right of this hatched region correspond to situations in which firing is prevented by too much inhibition. Note that firing is also absent above and to the left of the hatched region. For these parameters, the model enters depolarization block and ceases to fire, a problem that is even more severe for AMPAmediated, vs. NMDA-mediated, excitation (Deister et al., 2009).

The shape of the firing region suggests that disinhibition may be more robust than excitation as a mechanism for burst generation. This is because for most starting conditions, the system will remain in the firing regime even after a complete removal of inhibition. In contrast, NMDA conductance values must stay within a bounded region to support burst firing. Too much excitation and the system will move vertically outside the oscillating region and cease firing due to depolarization block.

This firing region was $\left[\mathrm{Mg}^{2+}\right]$ dependent (Figure 2E). Increasing $\mathrm{Mg}^{2+}$ RUNGE-KUTTA method concentrations reduces the negative slope conductance of the NMDA conductance, and the firing region becomes elongated. Under these conditions, the action of disinhibition and excitation are more similar in that removing too much inhibition will also drive the model into depolarization block and shut off bursting.
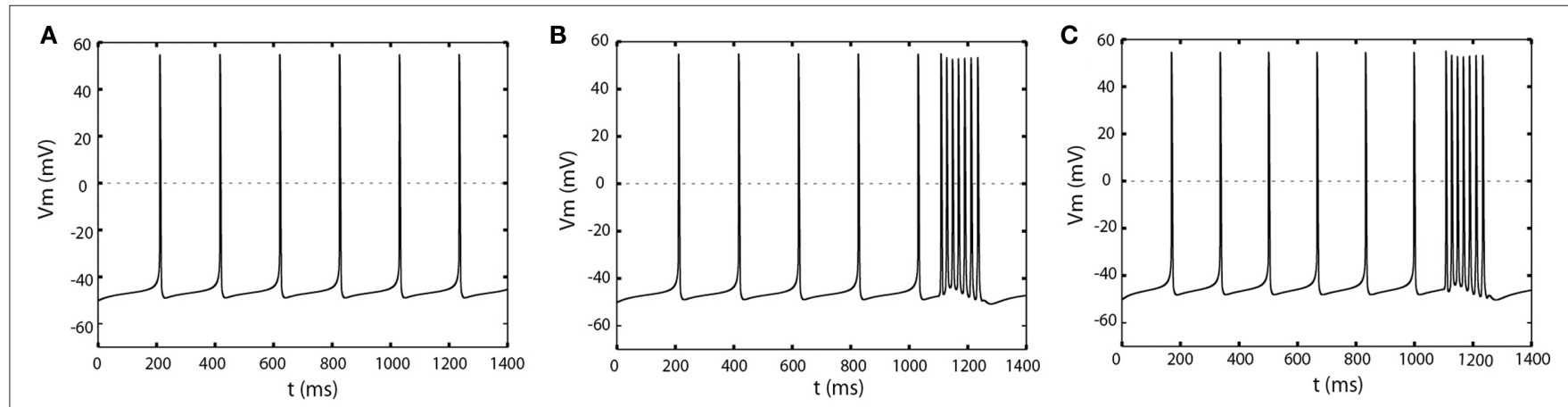

D

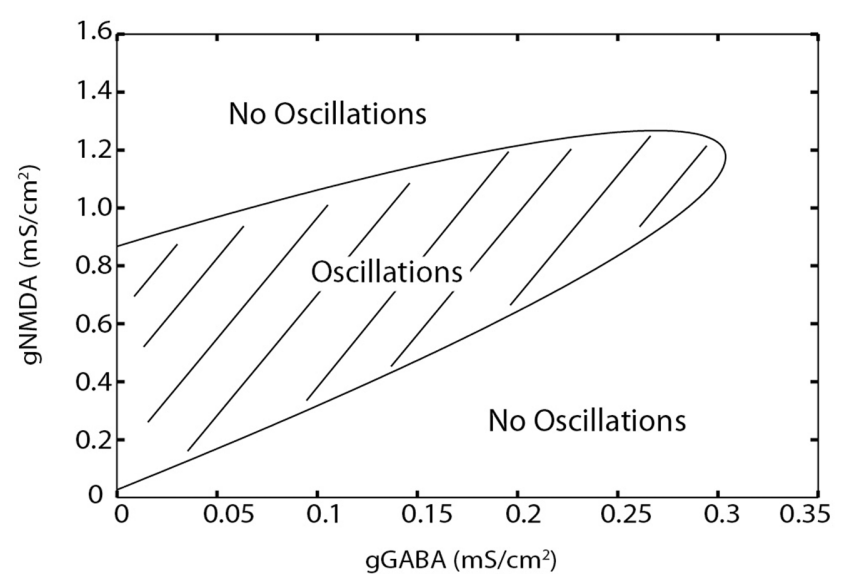

FIGURE 2 |The dopaminergic neuron can fire disinhibition bursts.

(A) Spontaneous, single-spiking in the absence of tonic input in a twocompartment model of the dopaminergic neuron. (B) Phasic activation of NMDA receptors (at $t=1100,150 \mathrm{~ms}$ ) produces a burst of action potentials. (C) The model exhibits single-spiking at similar frequencies as in (A) and can
E



produces bursts by disinhibition upon complete removal of tonic inhibition at $t=1100 \mathrm{~ms}$ for $150 \mathrm{~ms}$. (D) The NMDA and GABA A $_{\mathrm{A}}$ conductances at which repetitive spiking can be generated (hatched region) is computed by a two-point bifurcation diagram in AUTO (XPPAUT). (E) Magnesium dependence of the oscillatory region. 


\section{DIRECT PATHWAY}

After considering the single cell model in isolation, we investigated how disinhibition bursts could be driven by synaptic input patterns resulting from a network model of the basal ganglia based on the model of Humphries et al. (2006; see Materials and Methods). We first considered the influence of the D1STR-SNpr-SNpc ("direct") pathway. All connections other than the D1STR-SNpr, SNpr-SNpc, STN-SNpr, and STN-SNpc were disabled. Neurons in D1STR received direct current injection intended to model a burst of cortical input. Outputs from the network model were read into XPPaut and incremented their respective conductance equations by $\delta$. These values were estimated such that the mean conductance was approximately $g_{\mathrm{GABAA}}=0.14 \mathrm{~ms} / \mathrm{cm}^{2}$ and $g_{\mathrm{NMDA}}=0.28 \mathrm{~ms} / \mathrm{cm}^{2}$. An example is shown in Figure 3. Stimulation of the striatum evoked a burst in the dopaminergic neuron. The number of spikes in that burst was dependent on the length of time in which the striatum was stimulated (Figures 4B,C; 30-150 ms). The intra-burst firing frequency, however, was unaffected (Figure 4A).


Spike train data is input into Kuznetsov model
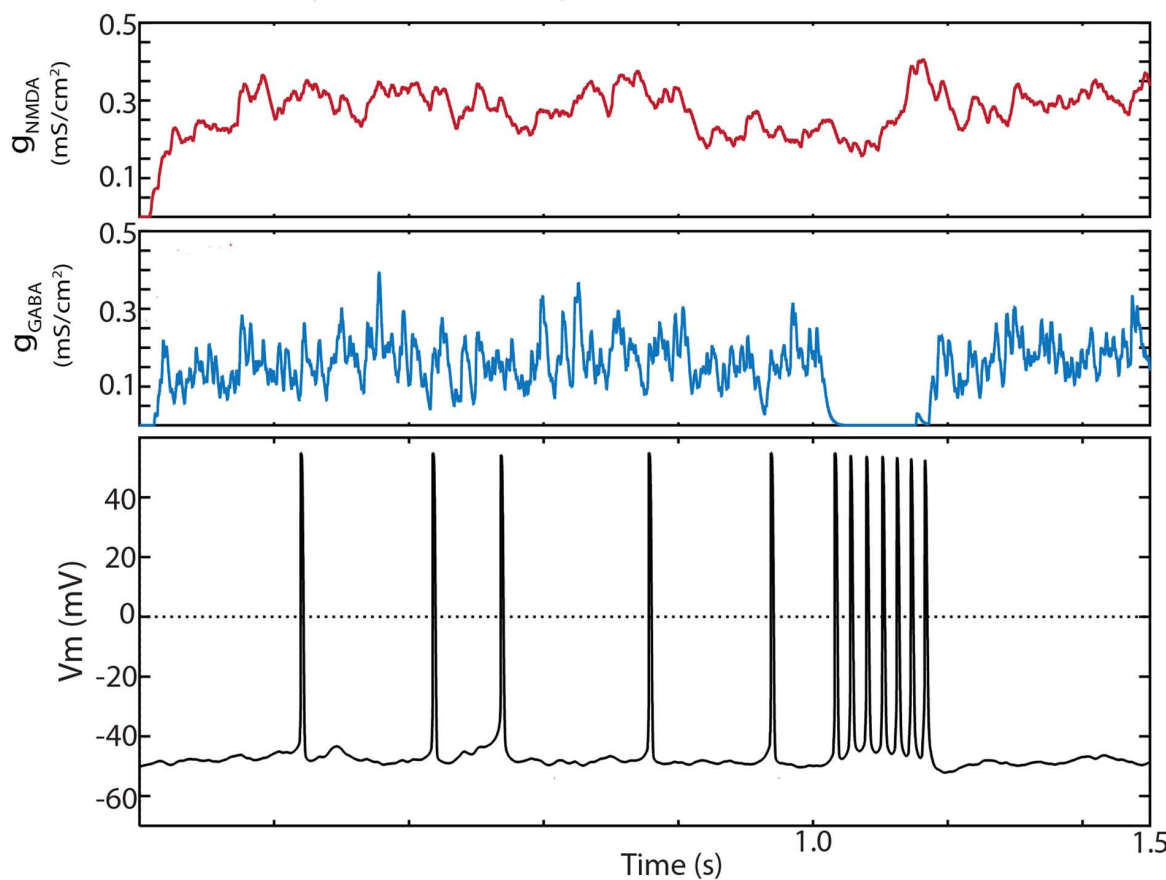

FIGURE 3 | Disinhibition bursts can be evoked by striatal stimulation in the reduced Humphries et al. (2006) model. Raster plots for the 64 neurons in the D1 striatum, STN and SNpr. The only connections present in the reduced model were D1 striatum to SNpr, STN-SNpc, STN-SNpr, and SNpr-SNpc. At $1 \mathrm{~s}$ all 64 D1 striatal projection neurons were activated by current injection for a period of $150 \mathrm{~ms}$. The simulation was run for a total of
$10 \mathrm{~s}$. The input to a random SNpc neuron was captured, saved to a file, and read in by the modified Kuznetsov et al. (2006) model. The synaptic conductances and resulting trace are shown below. Conductances were initially set at zero. The dopaminergic neuron model fired single spikes at approximately $5 \mathrm{~Hz}$ and exhibited a burst of action potentials during the period of striatal stimulation. 
What percentage of the medium spiny neurons must become activated to sufficiently inhibit the SNpr neurons to cause disinhibition bursting in the SNpc? In the previous simulations, all of the medium spiny neurons in the D1STR were stimulated at $t=1000 \mathrm{~ms}$. Changing the probability that a D1STR neuron was stimulated had a large effect on the intra-burst firing frequency and the number of spikes in the burst (Figure 5). The probability for which an individual D1STR neuron was stimulated was set to 0.03 ( 1 of 64 cells activated), $0.1,0.25,0.4,0.6,0.8$, or 1.0. A high frequency burst of a significant number of spikes was generated in response to a stimulation of only a few neurons (Figure 5). Thus, even weak striatal stimulation can evoke bursts in dopaminergic neurons.
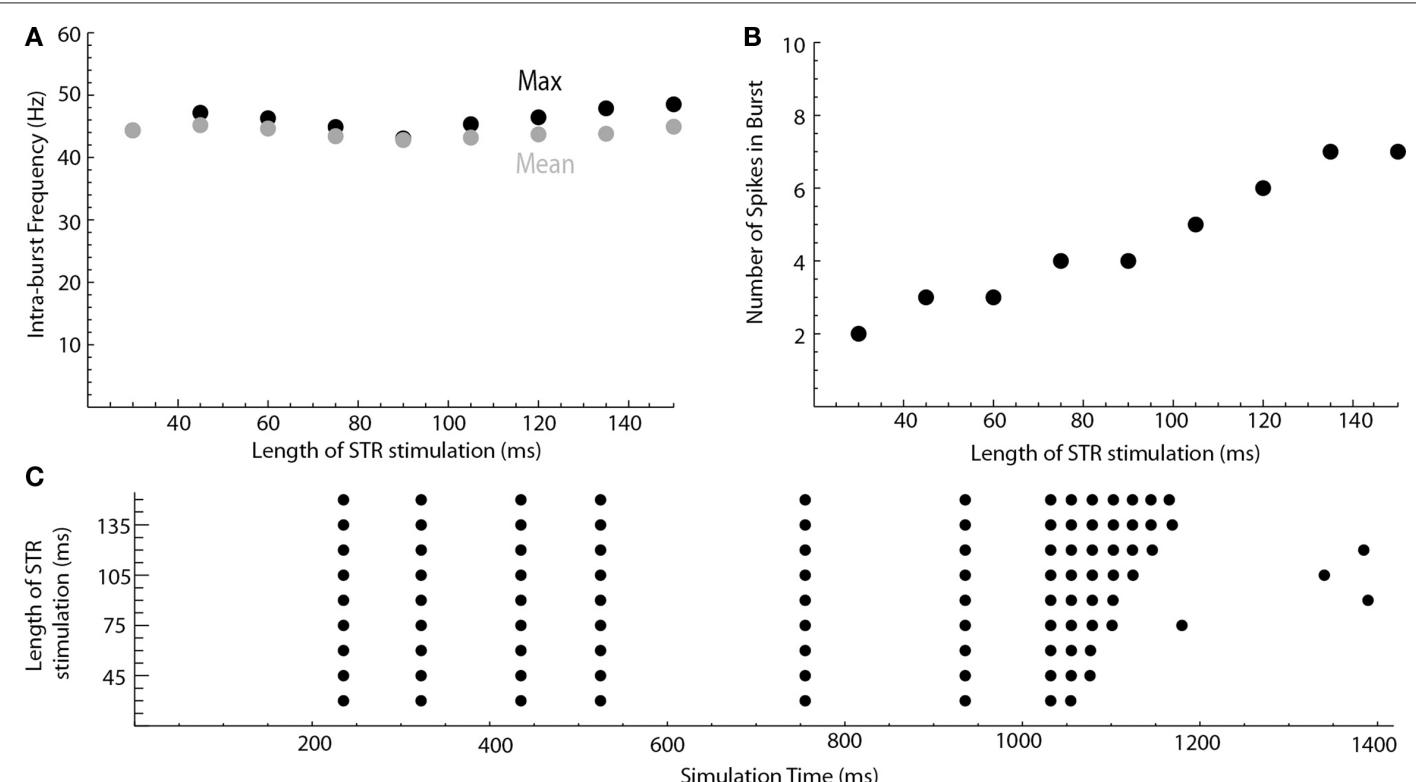

FIGURE 4 | Length of striatal stimulation controls the number of spikes in the disinhibition burst but not the intra-burst firing frequency. (A) Shows the intra-burst firing frequency of the disinhibition burst (max. black, mean gray). (B) Shows the number of spikes in the burst. A raster plot of the results is shown in (C).
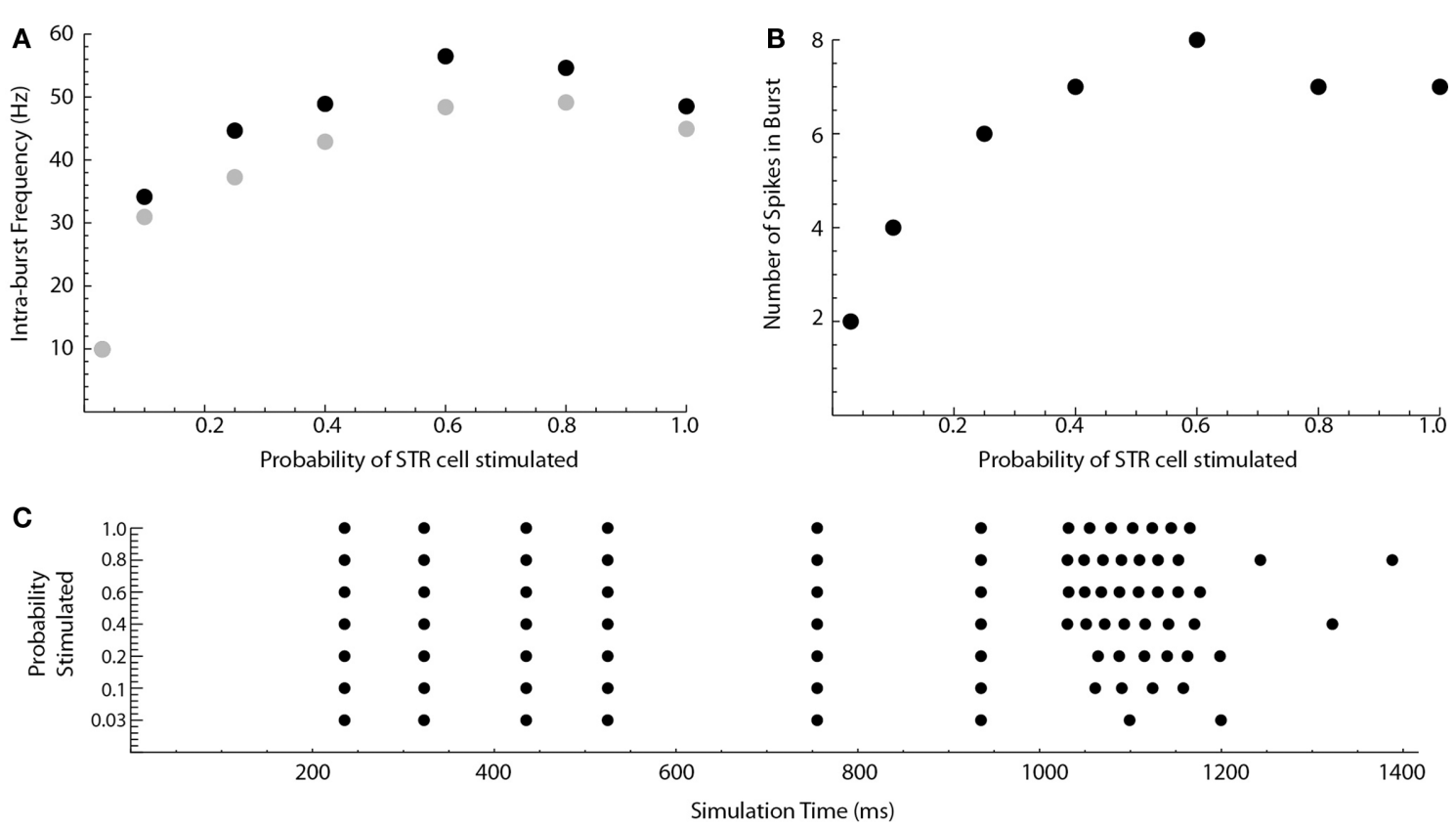

FIGURE 5 [Weak striatal stimulation can cause disinhibition bursts. The probability that a striatal cell will be stimulated at $t=1000$ ms in the reduced Humphries model is varied. (A) Shows the intra-burst firing frequency of the disinhibition burst (max. black, mean gray). (B) Shows the number of spikes in the burst. A raster plot of the results is shown in (C). 


\section{INDIRECT AND DIRECT PATHWAYS}

Do dopaminergic neurons still evoke a burst when the indirect pathway is included in the model? The connections between GPSNpc, GP-STN, STN-GP, GP-SNpr, and STN-SNpr were enabled. The constant current parameter for each cell type was modified such that the firing rates of the STN and SNpr were similar to the simulations presented in the previous section. Bursts were still evoked upon stimulation of all striatal neurons (Figure 6). Note that the inhibition is not completely removed during striatal stimulation. When the above indirect pathway connections were enabled in the network model, SNpc dopaminergic neurons received tonic inhibitory input from two sources: SNpr and GP. In the simulations shown in Figure 6, only SNpr neurons were inhibited upon D1STR stimulation. Thus, disinhibition need not be an all-or-none phenomenon (e.g., Lobb et al., 2011).

D1STR neurons projecting to the SNpr give off collaterals that terminate in the GP (Kawaguchi et al., 1990; Parent et al., 1995). This connection was added to the network model. Disinhibition bursting was enhanced by inclusion of the D1STR-GP connection (Figure 7). An example is shown in Figure 7A for a D1STR-GP weight of -1.0. Near-maximal frequency bursting could be obtained with a connection strength of only 0.2 (in comparison with other connections, which have a weight of \pm 1.0 ). The enhancement in SNpc activity is due to an inhibition of both GP and SNpr neurons (disinhibiting SNpc neurons) and a disinhibition of the STN through the GP (strongly exciting SNpc neurons). This suggests that striatal activation is a robust means by which bursts can be generated by SNpc dopaminergic neurons.

\section{DISCUSSION}

In this paper, we used computer simulations with network-generated time-varying conductances to explore disinhibition bursting in the basal ganglia. We first showed that the two-compartment single cell model of the dopaminergic neuron maintained its ability to fire trains of action potentials when tonic NMDA and GABA conductances were applied. We then used a bifurcation analysis to map out the combinations of NMDA and GABA conductances for which the model was able to produce sustained trains of action potentials. As expected, increasing inhibition or lowering excitation shifts the dopaminergic neuron into the non-oscillatory region, resulting in a pause in spiking (as seen in Figure 3A of Lobb et al., 2010). The bifurcation analysis revealed that large increases in excitation can also cause the dopaminergic neuron to cross into the non-oscillatory region, with the cessation of spiking due to a depolarization block of sodium mediated action potentials. In contrast, for moderate levels of the NMDA conductance, spiking was maintained even after complete removal of inhibition.

Disinhibition bursts could be evoked through the D1STR-SNprSNpc pathway by phasic activation of the D1STR. The duration of the striatal stimulation is an important determinant of the number of spikes in the burst, but has little effect on its intra-burst firing frequency. Striatal inhibition of the SNpr is powerful and thus only a small percentage of striatal cells need to be activated to evoke a burst in dopaminergic neurons.

Tonic inhibition onto dopaminergic neurons is a combination of inputs from GP and SNpr. In our simulations, the firing rate of the GP and SNpr were similar, and thus silencing of the SNpr would only remove that percentage of the tonic inhibition. The burst in $\mathrm{SNpc}$ neurons is affected by the percentage of tonic inhibition that is removed by a disinhibitory stimulus such as striatal activation; specifically, increased removal of inhibition increases the intraburst firing frequency of the disinhibition burst (Lobb et al., 2011).

Disinhibition of the SNpc was enhanced by including a collateral from D1STR to the GP. This was due to an inhibition of the GP (thereby disinhibiting $\mathrm{SNpc}$ ) and subsequent disinhibition of the subthalamic nucleus (thereby exciting the SNpc). Another interesting question that

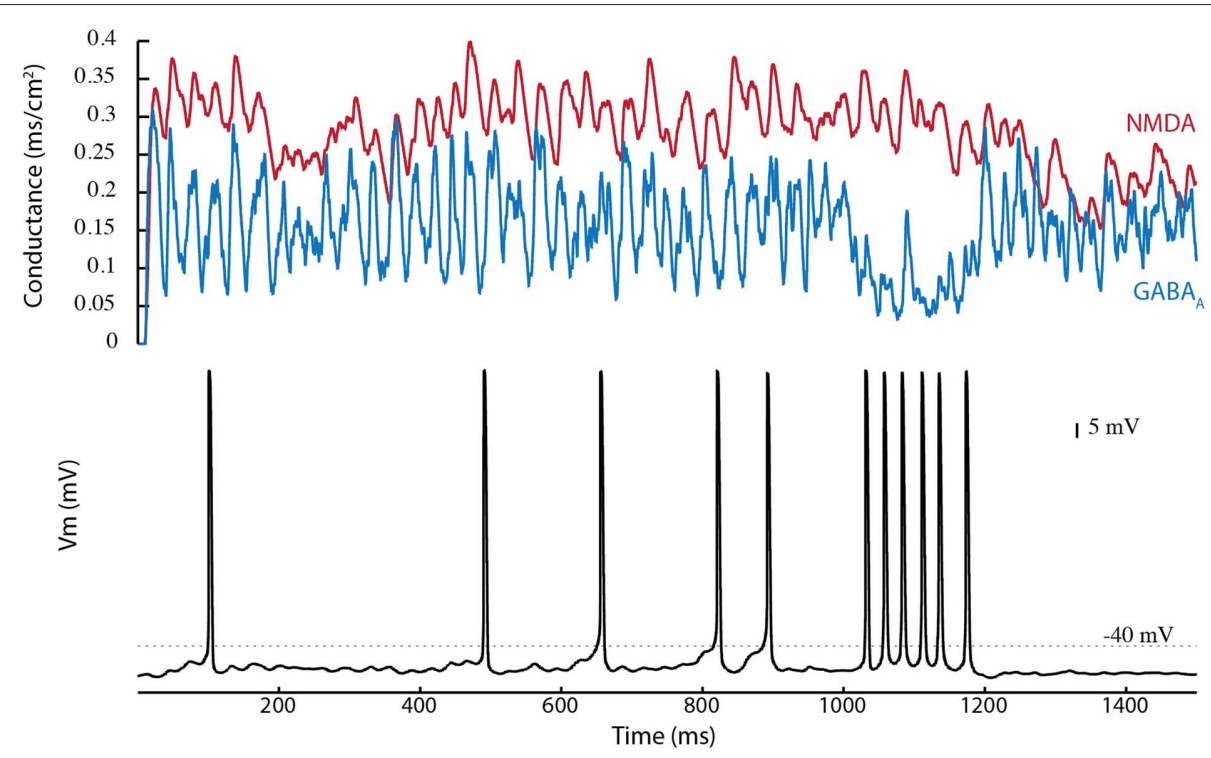

FIGURE 6 |Tonic inhibition by GP reduces direct pathway mediated disinhibition bursting in the Humphries et al. (2006) model. In the Humphries model, tonic inhibition is provided by both the GP and SNpr. All connections are added except the D1STR-GP connection. Since GP and SNpr have similar firing rates, silencing of the SNpr by striatal stimulation removes approximately half of the inhibition at $t=1000$ ms. Disinhibition bursts can still be produced. 


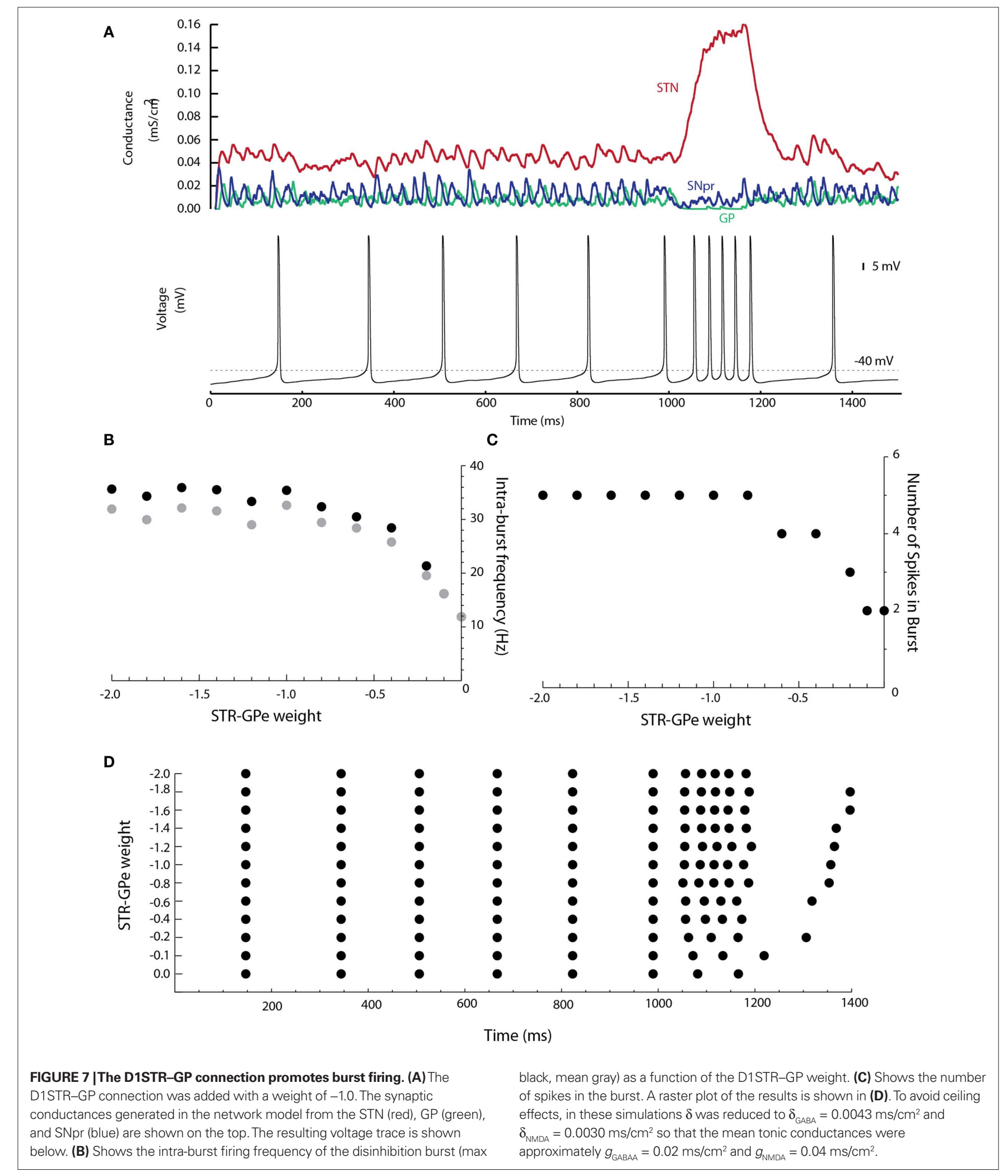

arises from these simulations is what the net effect of striatal stimulation will be on the SNpr and its disinhibition. The SNpr is inhibited by striatal activation but excited through the STN. The GP may also disinhibit the SNpr depending on whether striatopallidal inhibition is stronger than recurrent pallidosubthalamic excitation. In these simulations, the net effect on the SNpr is an inhibition. To achieve effective disinhibition, the SNpr excitation through the indirect pathway should be minimized, e.g., by increasing the weight of D1STR-SNpr synapses. 
In this model, only some of the basic features of these connections are implemented. Many features, such as a high degree of convergence from one nucleus onto another, the action of neuromodulators, synaptic enhancement or depression, and the presence of correlated activity within different nuclei are not included. We anticipate that factors such as these may play a large role in determining the weights of each connection and thus could have a major impact on bursting. Similarly, we have focused on stimulation of the D1-containing medium spiny neurons. Cortical activation of D2-containing medium spiny neurons may also evoke bursting in dopaminergic neurons similar to our results above. Finally, cortical activation may also evoke strong excitation in subthalamic neurons via the hyperdirect pathway. However, unlike striatal stimulation, activity in the GP and SNpr would be increased, thereby counteracting the

\section{REFERENCES}

Blythe, S. N., Atherton, J. F., and Bevan, M. D. (2007). Synaptic activation of dendritic AMPA and NMDA receptors generates transient high-frequency firing in substantia nigra dopamine neurons in vitro. J. Neurophysiol. 97, 2837-2850.

Blythe, S. N., Wokosin, D., Atherton, J. F., and Bevan, M. D. (2009). Cellular mechanisms underlying burst firing in substantia nigra dopamine neurons. $J$. Neurosci. 29, 15531-15541.

Brazhnik, E., Shah, F., and Tepper, J. M. (2008). GABAergic afferents activate both GABAA and GABAB receptors in mouse substantia nigra dopaminergic neurons in vivo. J. Neurosci. 28 , 10386-10398.

Charlety, P. J., Grenhoff, J., Chergui, K., De la Chapelle, B., Buda, M., Svensson, T. H., and Chouvet, G. (1991). Burst firing of mesencephalic dopamine neurons is inhibited by somatodendritic application of kynurenate. Acta Physiol. Scand. 142, 105-112.

Chergui, K., Akaoka, H., Charléty, P. J., Saunier, C. F., Buda, M., and Chouvet, G. (1994). Subthalamic nucleus modulates burst firing of nigra dopamine neurons via NMDA receptors. Neuroreport 5, 1185-1188.

Chergui, K., Charlety, P. J., Akaoka, H., Saunier, C. F., Brunet, J. L., Buda, M., Svensson, T.H., and Chouvet, G. (1993). Tonic activation of NMDA receptors causes spontaneous burst discharge of rat midbrain dopamine neurons in vivo. Eur. J. Neurosci. 5, 137-144.

Christoffersen, C. L., and Meltzer, L. T. (1995). Evidence for $N$-methyl-Daspartate and AMPA subtypes of the glutamate receptor on substantia nigra dopamine neurons: possible preferential role for $N$-methyl-D-aspartate receptors. Neuroscience 67, 373-381.

Deister, C. A., Teagarden, M., Wilson, C. J., and Paladini, C. A. (2009). An intrinsic neuronal oscillator underlies dopaminergic neuron bursting. $J$. Neurosci. 29, 15888-15897.
Ermentrout, B. (2002). Simulating, Analyzing, and Animating Dynamical Systems: A Guide to Xppaut for Researchers and Students. Philadelphia, PA: Society for Industrial and Applied Mathematics.

Gerfen, C. R. (1984). The neostriatal mosaic: compartmentalization of corticostriatal input and striatonigral output systems. Nature 311, 461-464.

Grace, A. A., and Bunney, B. S. (1984). The control of firing pattern in nigral dopamine neurons: burst firing. $J$. Neurosci. 4, 2877-2890.

Grace, A. A., and Bunney, B. S. (1985). Opposing effects of striatonigral feedback pathways on midbrain dopamine cell activity. Brain Res. 333, 271-284.

Grace, A. A., and Onn, S. P. (1989). Morphology and electrophysiological properties of immunocytochemically identified rat dopamineneurons recorded in vitro. J. Neurosci. 9, 3463-3481.

Grenhoff, J., Aston-Jones, G., and Svensson, T. H. (1986). Nicotinic effects on the firing pattern of midbrain dopamine neurons. Acta Physiol. Scand. 128, 351-358.

Guzman, J. N., Sánchez-Padilla, J., Chan, C. S., and Surmeier, D. J. (2009). Robust pacemaking in substantia nigra dopaminergic neurons. $J$. Neurosci. 29, 11011-11019.

Horvitz, J. C., Stewart, T., and Jacobs, B. L. (1997). Burst activity of ventral tegmental dopamine neurons is elicited by sensory stimuli in the awake cat. Brain Res. 759, 251-258.

Humphries, M. D., Stewart, R. D., and Gurney, K.N. (2006).A physiologically plausible model of action selection and oscillatory activity in the basal ganglia. J. Neurosci. 26, 12921-12942.

Jin, X., and Costa, R. M. (2010). Start/stop signals emerge in nigrostriatal circuits during sequence learning. Nature 466, 457-462.

Kawaguchi, Y., Wilson, C. J., and Emson, P. C. (1990). Projection subtypes of

STN-mediated excitation, and reducing the frequency of the ensuing burst.

\section{CONCLUSION}

In summary, we investigated the high conductance state of the dopaminergic neuron and show that disinhibition bursts can be produced through the D1STR-SNpr-SNpc circuit. Inclusion of the indirect pathway actually promotes disinhibition bursting. Together, these results suggest that striatal activation of the $\mathrm{SNpc}$ is not just a feasible mechanism for evoking disinhibition bursts. Instead, it may provide a reliable and robust means of evoking bursts in dopaminergic neurons.

\section{ACKNOWLEDGMENTS}

This work was supported by NIH grants MH079276 to Carlos A. Paladini, and MH084494 to Collin J. Lobb.

rat neostriatal matrix cells revealed by intracellular injection of biocytin. J. Neurosci. 10, 3421-3438.

Khaliq, Z. M., and Bean, B. P. (2010). Pacemaking in dopaminergic ventral tegmental area neurons: depolarizing drive from background and voltagedependent sodium conductances. $J$. Neurosci. 30, 7401-7413.

Kita, T., Kita, H., and Kitai, S. T. (1986) Electrical membrane properties of rat substantia nigra compacta neurons in an in vitro slice preparation. Brain Res. 372, 21-30.

Kuznetsov, A. S., Kopell, N. J., and Wilson, C. J. (2006). Transient high-frequency firing in a coupledoscillator model of the mesencephalic dopaminergic neuron. $J$. Neurophysiol. 95, 932-947.

Lobb, C. J., Wilson, C. J., and Paladini, C. A. (2010). A dynamic role for GABA receptors on the firing pattern of midbrain dopaminergic neurons. J. Neurophysiol. 104, 403-413.

Lobb, C. J., Wilson, C. J., and Paladini, C. A. (2011). High frequency, short latency disinhibition bursting of midbrain dopaminergic neurons. J. Neurophysiol. doi: 10.1152/ jn.01076.2010. [Epub ahead of print].

Morikawa, H., Khodakhah, K., and Williams, J. T. (2003). Two intracellular pathways mediate metabotropic glutamate receptor-induced $\mathrm{Ca} 2+$ mobilization in dopamine neurons. J. Neurosci. 23, 149-157.

Overton, P., and Clark, D. (1992). Iontophoretically administered drugs acting at the $N$-methyl-D-aspartate receptor modulate burst firing in A9 dopamine neurons in the rat. Synapse 10, 131-140.

Paladini, C. A., and Tepper, J. M. (1999). $\operatorname{GABA}(\mathrm{A})$ and $\mathrm{GABA}(\mathrm{B})$ antagonists differentially affect the firing pattern of substantia nigra dopaminergic neurons in vivo. Synapse 32, 165-176.
Parent, A., Charara, A., and Pinault, D. (1995). Single striatofugal axons arborizing in both pallidal segments and in the substantia nigra in primates. Brain Res. 698, 280-284.

Redgrave, P., Prescott, T. J., and Gurney, K. (1990). Is the short-latency dopamine response too short to signal reward error? Trends Neurosci. 22, 146-161.

Schultz, W. (1998). Predictive reward signal of dopamine neurons. J. Neurophysiol. 80, 1-27.

Schultz, W., Dayan, P., and Montague P. R. (1997).A neural substrate of prediction and reward. Science 275, 1593-1599.

Smith, I. D., and Grace, A. A. (1992). Role of the subthalamic nucleus in the regulation of nigral dopamine neuron activity. Synapse 12, 287-303.

Tepper, J. M., and Lee, C. R. (2007). GABAergic control of substantia nigra dopaminergic neurons. Prog. Brain Res. 160, 189-208.

Wilson, C. J., and Callaway, J. C. (2000). Coupled oscillator model of the dopaminergic neuron of the substantia nigra. J. Neurophysiol. 83, 3084-3100.

Conflict of Interest Statement: The authors declare that the research was conducted in the absence of any commercial or financial relationships that could be construed as a potential conflict of interest.

Received: 26 January 2011; accepted: 26 April 2011; published online:10 May 2011. Citation: Lobb CJ, Troyer TW, Wilson CJ and Paladini CA (2011) Disinhibition bursting of dopaminergic neurons. Front. Syst. Neurosci. 5:25. doi: 10.3389/ fnsys.2011.00025

Copyright ( $(2011$ Lobb, Troyer, Wilson and Paladini. This is an open-access article subject to a non-exclusive license between the authors and Frontiers Media SA, which permits use, distribution and reproduction in other forums, provided the original authors and source are credited and other Frontiers conditions are complied with. 